\title{
香豆素-咪唑类荧光染料的设计与研究
}

\author{
后际挺 $*, a, c$ 王冰雅 ${ }^{a}$ 李 坤 ${ }^{b}$ 汪 珊*,a 余孝其 $*, b$ \\ ( ${ }^{a}$ 信阳师范学院化学化工学院 河南信阳 464000) \\ ( ${ }^{b}$ 四川大学化学学院 成都 610064) \\ ( ${ }^{c}$ 湖北工程学院化学与材料科学学院 湖北孝感 432000)
}

\begin{abstract}
摘要 合成了不同给电子取代基(羟基、丁氧基、二乙基氨基等)的菲并 $[9,10-d]$ 咪唑(CA1 CA6)或 4,5-二苯基咪唑 (CB1 C CB6)修饰的香豆素衍生物, 初步考察了它们的溶液发光和固体发光现象. 研究表明, 当香豆素取代基为氨基时, 化合物在二氯甲烷中的苂光较强，而羟基取代、丁氧基取代或者无取代的衍生物在二氯甲烷中的苂光都很弱，而菲并 $[9,10-d]$ 咪唑修饰的衍生物 CA1 C CA5 的溶液荧光要比 4,5-二苯基取代咪唑修饰的衍生物 CB1 CB5 的溶液强. 另外, 染料分子的分子内氢键强度及咪唑基-香豆素环间二面角大小都会对染料分子的发光性能产生影响.
\end{abstract}

关键词 香豆素; 咪唑; 分子内氢键; 苂光

\section{Design and Study of Coumarin-Imidazol Dyes}

\author{
Hou, Jiting*, $\quad$ Wang, Bingya $^{a} \quad$ Li, Kun ${ }^{b} \quad$ Wang, Shan ${ }^{*, a} \quad$ Yu, Xiaoqi ${ }^{*, b}$ \\ ( ${ }^{a}$ College of Chemistry and Chemical Engineering, Xinyang Normal University, Xinyang, He'nan 464000) \\ ( ${ }^{b}$ College of Chemistry, Sichuan University, Chengdu 610064) \\ ( ${ }^{c}$ School of Chemistry and Materials Science, Hubei Engineering University, Xiaogan, Hubei 432000)
}

\begin{abstract}
A series of phenanthro[9,10- $d]$ imidazole $(\mathbf{C A 1} \sim \mathbf{C A 6})$ or 4,5-diphenyl imidazole $(\mathbf{C B 1} \sim \mathbf{C B 6})$ modified coumarin derivatives with different electron-donating substitutes were synthesized, and their fluorescences in organic solvent and solid were primarily explored. The results showed that the amino substituents in coumarin skelton enabled strong fluorescence in dichloromethane, while hydroxyl-, butoxyl-, or non-substituted derivatives showed very weak emission in dichloromethane. Meanwhile, phenanthro[9,10- $d$ ] imidazole modified coumarin dervatives CA1 $\sim$ CA5 exhibited stronger fluorescence than that of 4,5-diphenyl imidazole modified ones. Additionally, the strenghts of intramolecular hydrogen bond as well as the dihedral angles of the imidazole moiety and coumarin ring affected the optical properties of these dyes.

Keywords coumarin; imidazole; intramolecular hydrogen bond; fluorescence
\end{abstract}

近几十年来，苂光技术发展成熟，苂光染料的开发 也受到了广泛关注.一般来说, 在高浓度下, 荧光染料 分子会发生强烈的分子间作用，从而导致荧光淬灭，即 聚集诱导淬灭效应(ACQ), 因此, 传统的荧光分析手段 需要在稀溶液中进行 ${ }^{1]}$. 然而, 溶液苂光分析技术需要 经历样品制备等繁琐步骤, 且受浓度限制, 发光强度有 限, 导致其应用有很大的局限性. 因此, 研究者通过将 染料掺杂入基质中, 形成苂光染料的固溶物, 得到有机 固体发光材料. 这一类发光材料在有机发光二极管 (OLEDs)、有机固体激光器等领域中扮演着越来越重要
的角色 ${ }^{[2]}$. 然而，掺杂类固体发光材料的制备工艺对材 料的浓度要求非常高, 荧光物质的含量一般只有几个百 分比, 进而大大提高了生产成本并影响了发光效果 ${ }^{[2]}$. 于是，开发非掺杂类的固体发光材料受到了广泛关注. 近年来, 已经有大量基于有机小分子的固体发光材料见 诸报道, 比如聚集诱导发光(AIE)类荧光染料 ${ }^{[3,4]}$.

随着有机小分子固体荧光染料的发展, 研究者们也 开发了各种新型的大共轭体系作为苂光团骨架，反而对 于传统的苂光骨架关注很少, 如罗丹明、香豆素、花青 素等，因为这些传统的荧光团通常都有 $\mathrm{ACQ}$ 效应，不

\footnotetext{
*Corresponding authors. E-mail: houjiting2206@163.com; smallcoral@live.cn; xqyu@scu.edu.cn Received August 12, 2019; revised September 23, 2019; published online October 12, 2019.

Project supported by the National Natural Science Foundation of China (Nos. 21807029, 21877082), the Hubei Provincial Natural Science Foundation (No. 2018CFB264) and the Nanhu Scholars Program for Young Scholars of XYNU (Xinyang Normal University).

国家自然科学基金((Nos. 21807029, 21877082)、湖北省自然科学基金(No. 2018CFB264)、信阳师范学院南湖学者奖励计划资助项目.
} 
适用于固体菼光材料的制备 ${ }^{[5 \sim 11]}$. 近年来, 很多课题组 对传统的氟硼类 ${ }^{[12 ~ 19]}$ 、蒽类 ${ }^{[20 ~ 23]}$ 苂光染料进行结构改 造, 得到了很多具有优良固体苂光性能的材料, 但是对

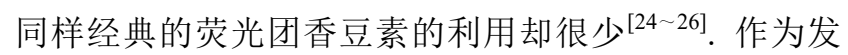
光效率和稳定性都很优异的一种荧光骨架, 香豆素还具 备一个很多苂光染料不具备的优势: 制备简单且产率较 高. 基于我们课题组前期在香豆素小分子荧光探针方面 的工作 ${ }^{[27 ~ 29]}$, 我们设想是否可以利用香豆素骨架制备 一系列在溶液状态和固体状态下同时具备优良发光性 能的染料, 从而满足不同应用需求.

如 Scheme 1 所示, 我们选择在香豆素的 3 位分别引 入菲并 [9,10- $d$ ]咪唑和 4,5-二苯基咪唑, 合成化合物 CA1 CA5 和 CB1 $\sim$ CB5, 并在 7 位引入供电子能力不 同的取代基, 以考察取代基对这一系列固体苂光的影 响. 咪唑基上的 NH 可以和香豆素 2 位的羰基形成分子 内氢键, 增加整个分子结构的刚性, 有利于大共轭体系 的稳定, 从而增加荧光分子发光效率. 和 CA1 CA5 相 比, CB1 C CB5 中的咪唑上两个苯环会以一定角度扭转, 从而避免相邻分子间的作用, 也会对发光效率起到促 进作用. 同时, 为了进一步研究分子内氢键对染料光学 性能的影响, 我们合成了 6-位取代的香豆素衍生物 CA6 和 CB6. 这两个化合物的咪唑环与香豆素环之间没有分 子内氢键的约束, 可以自由旋转, 因此可能会表现出与 3 位取代的染料截然不同的荧光性质.
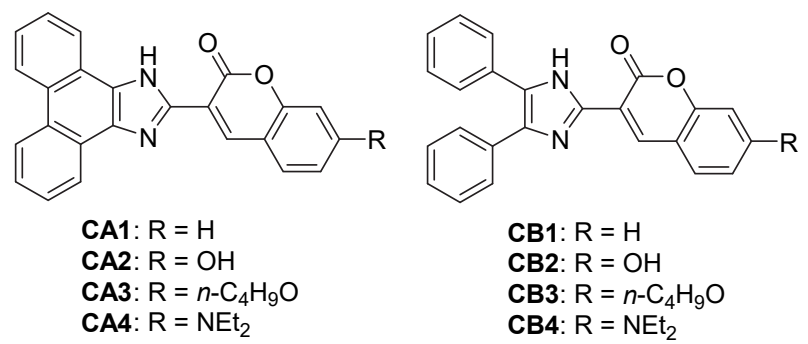

$$
\begin{aligned}
& \text { CB1: } \mathrm{R}=\mathrm{H} \\
& \text { CB2: } \mathrm{R}=\mathrm{OH} \\
& \text { CB3: } \mathrm{R}=n-\mathrm{C}_{4} \mathrm{H}_{9} \mathrm{O} \\
& \text { CB4: } \mathrm{R}=\mathrm{NEt}_{2}
\end{aligned}
$$
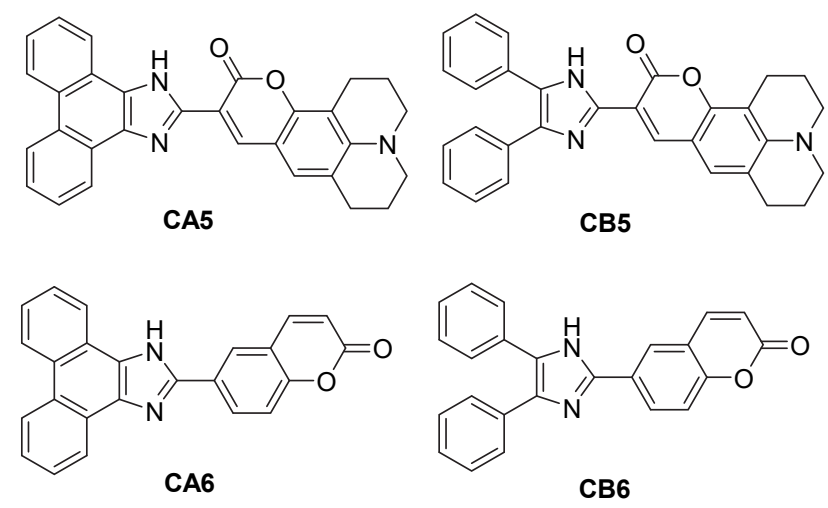

图式 1 香豆素-咪唑类荧光染料结构式 Scheme 1 Structures of coumarin-imidazole dyes

\section{1 结果与讨论}

\section{1 化合物的合成}

化合物 CA1 $\sim$ CA6 和 CB1 $\sim$ CB6 的合成如 Scheme 2 所示. 将香豆素醛中间体分别和 9,10-邻菲啰啉二酮或 者二苯乙二酮在过量无水醋酸铵存在下，一步反应得到 目标产物. 反应时间从 $2 \mathrm{~h}$ 到 $4 \mathrm{~h}$ 不等. 目标化合物的结 构均通过 ${ }^{1} \mathrm{H} N M R 、{ }^{13} \mathrm{C} N \mathrm{NMR}$ 和 HRMS 进行确证. 其中, 化合物 CA1、CB1、CB3、CA4、CB4 和 CB5 的晶体 结构获得了确认.

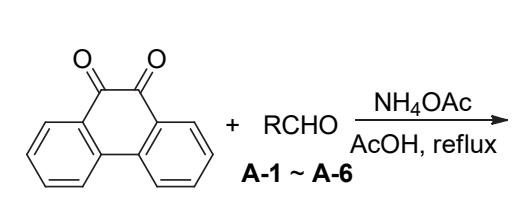<smiles>[R]c1nc2c3ccccc3c3ccccc3c2[nH]1</smiles>

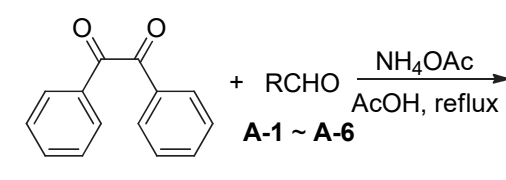

CA1 CA6

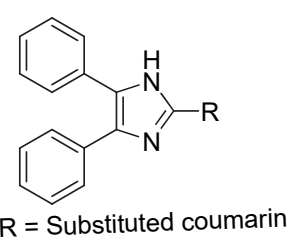

CB1 CB6<smiles>O=Cc1cc2ccccc2oc1=O</smiles>

A-1<smiles>COc1ccc2cc(C=O)c(=O)oc2c1</smiles>

A-2<smiles>CCCCOc1ccc2cc(C=O)c(=O)oc2c1</smiles>

A-3<smiles>CCN(CC)c1ccc2cc(CO)c(=O)oc2c1</smiles>

图式 2 中间体香豆素醛的结构及香豆素-咪唑类苂光染料的 合成路线

Scheme 2 Structures of coumarin aldehydes, and the synthetic route of coumarin-imidazole dyes

\section{2 化合物的光学性质分析}

首先研究了 $\mathrm{CA1} \sim \mathrm{CA6}$ 和 CB1 $\sim \mathrm{CB} 6$ 分别在二氯 甲烷溶液和粉末状态下的光学性质. 如表 1 所示, 在二 氯甲烷溶液中，随着 7 位取代基的供电子性增强，化合 物 CA1 到 CA5、CB1 到 CB5 的最大吸收波长分别向长 波处移动; 而在香豆素 7-位取代基不变时，菲并 [9,10- $d$ ] 咪唑修饰的化合物的最大吸收波长 $\left(\lambda_{\mathrm{abs}}\right)$ 要比二苯基咪 唑修饰的化合物的 $\lambda_{\mathrm{abs}}$ 长 $11 \sim 12 \mathrm{~nm}$, 说明 7-位取代基 的供电子性和 3-位取代咪唑基的刚性都对香豆素染料 的共轭体系产生了影响. 比较有意思的是, 这一系列化 合物的 $\lambda_{\mathrm{abs}}$ 虽然呈规律性的变化, 但是它们在二氯甲烷 中的最大发射波长 $\lambda_{\mathrm{em}}$ 基本一致，均在 $505 \sim 511 \mathrm{~nm}$ 之 间波动，如表 1 和图 1 所示. 同时，这一系列化合物在二 
表 1 化合物 $\mathbf{C A 1} \sim \mathrm{CA5}$ 和 CB1 CB5 的光学数据

Table 1 Optical data of compounds CA1 CA5 and CB1 $\sim$ CB5

\begin{tabular}{|c|c|c|c|c|c|c|}
\hline \multirow{2}{*}{ Compd. } & \multicolumn{4}{|c|}{$\mathrm{CH}_{2} \mathrm{Cl}_{2}$} & \multicolumn{2}{|c|}{ Solid } \\
\hline & $\lambda_{\mathrm{abs}} / \mathrm{nm}$ & $\lambda_{\mathrm{em}} / \mathrm{nm}$ & $\Phi_{\mathrm{f}}^{a}$ & $\varepsilon /\left(10^{4} \mathrm{~L} \cdot \mathrm{mol}^{-1} \cdot \mathrm{cm}^{-1}\right)$ & $\lambda_{\mathrm{em}} / \mathrm{nm}$ & $\Phi_{\mathrm{s}}^{b} / \%$ \\
\hline CA1 & 412 & 509 & 0.014 & 2.8 & 547 & 4.32 \\
\hline CB1 & 400 & 511 & 0.002 & 2.0 & 571 & 1.83 \\
\hline CA2 & 413 & 506 & 0.049 & 4.9 & 572 & $-^{c}$ \\
\hline CB2 & 402 & 507 & 0.003 & 3.8 & 530 & $-^{c}$ \\
\hline CA3 & 418 & 507 & 0.041 & 4.0 & 548 & 5.3 \\
\hline CB3 & 406 & 508 & 0.004 & 3.1 & 528 & 3.51 \\
\hline CA4 & 450 & 506 & 0.46 & 5.0 & 630 & 4.19 \\
\hline CB4 & 439 & 505 & 0.28 & 4.1 & 550 & 0.67 \\
\hline CA5 & 465 & 511 & 0.60 & 5.5 & 615 & 5.9 \\
\hline CB5 & 453 & 509 & 0.44 & 1.2 & 600 & 2.84 \\
\hline
\end{tabular}

${ }^{a}$ The quantum yield $\Phi_{\mathrm{f}}$ was measured using fluorescein in $0.1 \mathrm{~mol} / \mathrm{L} \mathrm{NaOH}$ solution as a standard $\left(\Phi_{\mathrm{f}}=0.85\right) .{ }^{b}$ The solid quantum yield $\Phi_{\mathrm{s}}$ is the absolute quantum yield. ${ }^{c}$ The solid quantum yield was too low to be detected.
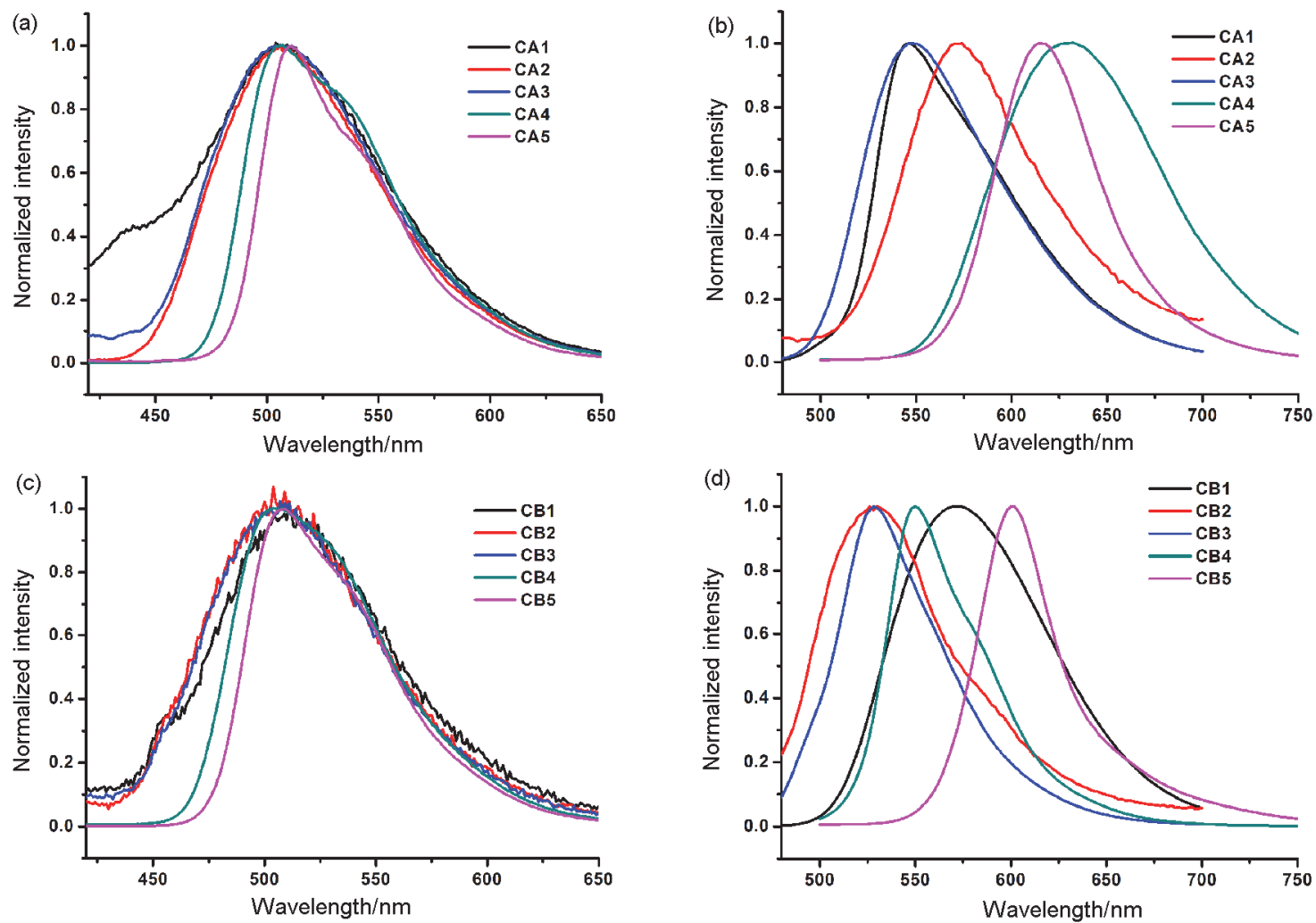

图 1 化合物 $\mathbf{C A 1} \sim \mathbf{C A 5}$ 和 $\mathbf{C B 1} \sim \mathbf{C B 5}$ 分别在二氯甲烷溶液中 $(\mathrm{a}$ 和 $\mathrm{c})$ 和粉末状态下 $(\mathrm{b}$ 和 $\mathrm{d})$ 的苂光光谱

Figure 1 Fluorescence spectra in $\mathrm{CH}_{2} \mathrm{Cl}_{2}$ and solid emission of $\mathbf{C A 1} \sim \mathbf{C A 5}$ (a and b) and $\mathbf{C B 1} \sim \mathbf{C B 5}$ (c and d)

氯甲烷中的荧光量子产率 $\Phi_{\mathrm{f}}$ 的变化规律与 $\lambda_{\mathrm{abs}}$ 的变化规 律基本一致: 取代基供电子能力越强, 量子产率越高; 结构刚性越大，量子产率越高. 其中，化合物 $\mathbf{C B 1} \sim$ CB3 基本是无荧光的. 我们猜测二苯基咪唑中的苯环自 由旋转会消耗荧光分子激发态的能量, 从而导致苂光淬 灭. 同时, 6 位取代的的 CA6 和 CB6 没有苂光, 可能是 因为 6 位的咪唑平面和香豆素平面之间没有氢键固定, 可以自由旋转, 从而消耗了化合物激发态的能量, 引起

\section{苂光淬灭.}

随后，考察了这一系列化合物在甲醇溶剂中的紫外 吸收光谱和苂光发射光谱. 相比于二氯甲烷溶液，同一 种化合物在甲醇中的吸收有微小蓝移，而发射波长却有 微小红移, 说明溶剂极性对这一系列化合物的基态和激 发态均有一定影响.

最后考察了这一系列化合物的固体荧光. 从表 1 和 图 2 中可以看出, 和它们的溶液苂光最大发射波长相差 
无几不同, CA1 CA5 和 CB1 CB5 的固体苂光最大发 射峰的位置差异十分明显, 并没有随着取代基供电子能 力的增强而出现规律性地红移, 说明取代基的给电子能 力在这一系列染料的固体发光中不是决定性影响因素. 另外, 除了 CB1 的固体苂光发射波长比 CA1 的红移 24 $\mathrm{nm}, \mathbf{C A 2} \sim \mathbf{C B 5}$ 的发射波长均比 $\mathbf{C B 2} \sim \mathbf{C B 5}$ 的更长, 红 移值最多可以达到 $80 \mathrm{~nm}$ (CA4 和 CB4), 所以总体来说 CA 系列固体荧光发射波长更长. 我们认为这是因为菲 并 $[9,10-d]$ 咪唑的刚性比二苯基咪唑的刚性更强, 而刚 性的增强有利于固体荧光红移. 其中, 7-二乙基氨基香 豆素衍生的化合物 CA4 的固体荧光发射波长可以达到 $630 \mathrm{~nm}$. 对于 6-位修饰的 CA6 和 CB6, CA6 在 $550 \mathrm{~nm}$ 处有中等强度的发射, 而 CB6 却没有固体苂光, 可能是 因为 CA6 的刚性更强.
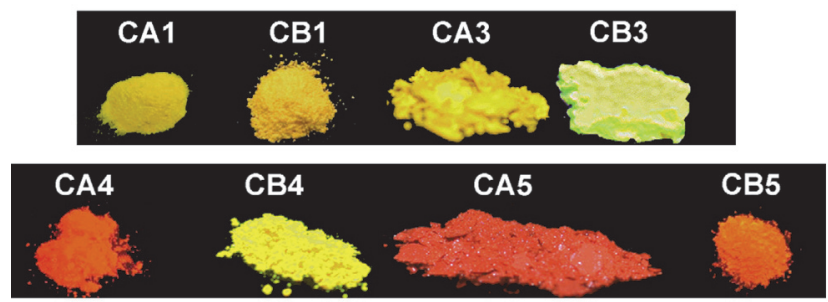

图 2 手提荧光灯下 $(365 \mathrm{~nm})$ 部分化合物的固体荧光

Figure 2 Solid fluorescence color of partial compounds under UV lamp (365 nm)

分析这一系列化合物的固体荧光量子产率 $\left(\Phi_{\mathrm{s}}\right)$, 可 以发现它们的 $\Phi_{\mathrm{s}}$ 都比较低, 最高是 CA5 的 $5.9 \%$, 而 CA2 和 CB2 的 $\Phi_{\mathrm{s}}$ 低至无法测量, 说明香豆素环上的取 代基对这一系列化合物的固体发光效率影响非常大. 另 外, 菲并 $[9,10-d]$ 咪唑取代的 CA 系列化合物的 $\Phi_{\mathrm{s}}$ 比 4,5二苯基咪唑取代的 $\mathrm{CB}$ 系列化合物的 $\Phi_{\mathrm{s}}$ 更高, 说明 4,5二苯基咪唑上两个苯环带来的非平面性对化合物的固 体发光效率并没有正效应。

\section{3 部分化合物的单晶结构分析}

为了了解导致这一系列化合物在固体发光中产生 差异的因素, 尝试了各种方法来培养单晶, 以探索这些 化合物的空间堆积模式和固体荧光之间的关系. 其中, CA1、CB1、CB4 和 CB5 的单晶通过缓慢挥发二氯甲 烷获得; CA4 的单晶在热的 DMSO 饱和溶液中逐渐冷却 析出; CB3 的单晶通过石油醚/DMSO 界面扩散法获得. 从图 3 的单晶结构以及表 2 的氢键数据可以看到, 当咪 坐基在香豆素 3-位时, 香豆素的 2 位 $\mathrm{C}=\mathrm{O}$ 确实可以和 咪唑的 $\mathrm{NH}$ 形成较强的 $\mathrm{N}-\mathrm{H} \cdots \mathrm{O}$ 氢键, 氢键键长在 $0.2123 \sim 0.2304 \mathrm{~nm}$ 之间. 由于氢键的存在, 3 -位修饰的 咪唑基与香豆素基本处于一个平面. 当 3 位修饰基为二 苯基咪唑时, 咪唑上的两个苯环以扭曲形式存在，这种 扭曲也阻止了相邻分子的靠近, 从而防止了聚集诱导的 荧光淬灭. 同时, 从单晶结构也可以看到，3-菲并 [9,10- $d]$ 咪唑修饰的结构平面性和刚性更强, 验证了前 面的猜测(除了 CA1 和 CB1).

从表 2 可以发现, CA1 和 CA4 的氢键键长 $\mathrm{H} \cdots \mathrm{A}$ 分 别比 CB1 和 CB4 的更短, 这可能意味着 CA1 CA5 中 的分子内氢键要比 $\mathrm{CB1} \sim \mathrm{CB} 5$ 中的更强. 结合前面所测 的 CA 系列溶液荧光量子产率要比 CB 系列高, 猜测分 子内氢键越强使得化合物溶液的苂光量子产率越高. 另 外, 比较 CB3、CB4 和 CB5 发现, 随着 7 位取代基给电 子能力的增强, $\mathrm{H} \cdots \mathrm{A}$ 键也增强, 固体苂光发射波长逐 渐红移, 说明取代基的给电子能力确实对化合物的结构 以及发光性能造成了影响. 然而, 对于 7 位无取代的化 合物 CB1，其 $\mathrm{H} \cdots \mathrm{A}$ 键长和 CB4 的几乎一样长，而固体 荧光发射波长则更长，进一步说明除了取代基的供电子 性，还有一些结构因素影响着这一系列化合物的固体发 光.

为了进一步探究影响这一系列化合物发光差异的 因素，随后比较了这 6 个化合物单晶中咪唑环和香豆素

表 2 各单晶氢键数据

Table 2 Data for hydrogen bonds in the single crystals

\begin{tabular}{|c|c|c|c|c|c|}
\hline Compd. & $\mathrm{D}-\mathrm{H} \cdots \mathrm{A}$ & $\mathrm{D}-\mathrm{H} / \mathrm{nm}$ & $\mathrm{H} \cdots \mathrm{A} / \mathrm{nm}$ & $\mathrm{D} \cdots \mathrm{A} / \mathrm{nm}$ & $\angle \mathrm{DHA} /\left(^{\circ}\right)$ \\
\hline CA1 & $\mathrm{N} 1-\mathrm{H} 1 \cdots \mathrm{O} 2$ & 0.0874 & 0.2135 & 0.2736 & 125.46 \\
\hline CB1 & $\mathrm{N} 2-\mathrm{H} 2 \cdots \mathrm{O} 2$ & 0.0861 & 0.2261 & 0.2783 & 119.02 \\
\hline CB3 & $\mathrm{N} 5-\mathrm{H} 5 \cdots \mathrm{O} 2$ & 0.0880 & 0.2304 & 0.2816 & 118.32 \\
\hline CA4 & $\mathrm{N} 1-\mathrm{H} 1 \cdots \mathrm{O} 2$ & 0.0860 & 0.2229 & 0.2752 & 119.17 \\
\hline CB4 & $\mathrm{N} 2-\mathrm{H} 2 \cdots \mathrm{O} 2$ & 0.0860 & 0.2260 & 0.2790 & 119.83 \\
\hline CB5 & $\mathrm{N} 3-\mathrm{H} 3 \cdots \mathrm{O} 2$ & 0.0860 & 0.2123 & 0.2692 & 123.24 \\
\hline
\end{tabular}



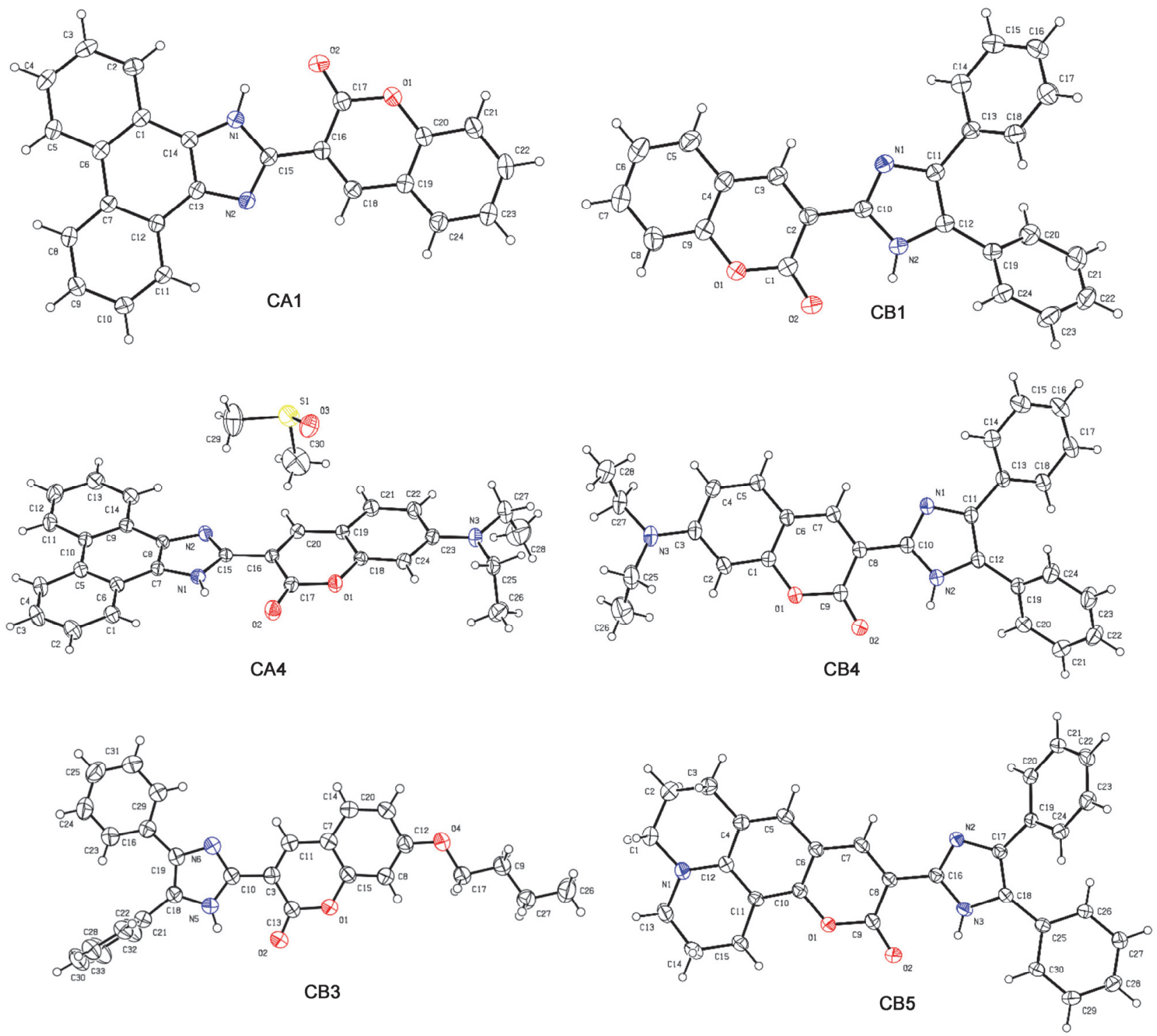

图 3 部分化合物的晶体结构示意图

Figure 3 X-ray crystallography of part of the compounds

环的二面角, 以比较它们的平面性差异. 从表 3 可以看 出, CA1 的二面角是 $4.79^{\circ}$, 比 CB1 的二面角 $\left(1.27^{\circ}\right)$ 明显 要大, 而 CA1 和 CB1 的固体荧光发射波长分别为 547 和 $571 \mathrm{~nm}$; 对比 CA4 和 CB4 发现, 两者的二面角分别 为 $5.59^{\circ}$ 和 $7.57^{\circ}$, 固体发射波长分别为 630 和 $550 \mathrm{~nm}$. 从这两组化合物的数据分析可以猜测, 在香豆素 7 位取 代基相同的情况下, 3 位修饰的咪唑环与香豆素平面的

\section{表 3 各单晶中咪唑环和香豆素环的二面角}

Table 3 Dihedral angles of the imidazole moiety and coumarin ring in each single crystal

\begin{tabular}{lcccccc}
\hline Compd. & CA1 & CB1 & CB3 & CA4 & CB4 & CB5 \\
\hline $\begin{array}{l}\text { Dihedral } \\
\text { angles } /\left(^{\circ}\right)\end{array}$ & 4.79 & 1.27 & 6.02 & 5.59 & 7.57 & 5.54 \\
\hline
\end{tabular}

二面角小, 导致固体苂光红移, 因为二面角小, 意味着 香豆素平面与咪唑环的共轭程度越高. 和 CB1 相比, CA1 的咪唑部分结构刚性更强, 但是由于其咪唑基和 香豆素环之间的扭转角略大，导致整个分子共轭程度相 对较弱, 最终使得 CA1 的固体荧光发射波长比 CB1 的 蓝移 $24 \mathrm{~nm}$. 因此, 在这一系列香豆素-咪唑体系的固体 发光中, 除了取代基供电子性之外，刚性和平面性也是 重要影响因素. 另外, 从氢键数据发现, 虽然 CB1 和 CB4 的氢键强度差不多, 但是 CB1 的固体苂光发射波 长要比 CB4 红移 $21 \mathrm{~nm}$, 这可能是由于 CB1 中咪唑基和 香豆素母核之间的二面角明显更小，即 CB1 中咪唑基 和香豆素的共轭程度更高. 


\section{2 结论}

合成了一系列基于传统荧光团香豆素的染料 CA1 $\sim$ CA6 和 CB1 $\sim$ CB6, 初步研究了它们的溶液苂光 和固体发光, 并对它们的单晶结构进行了初步分析. 研 究发现, 当香豆素 7 位取代基为氨基取代基时, 化合物 在二氯甲烷中的苂光较强, 而羟基取代、丁氧基取代或 者无取代的衍生物在二氯甲烷中的荧光都很弱; 另外, 菲并 $[9,10-d]$ 咪唑修饰的衍生物 CA1 CA6 的溶液荧光 要比 4,5-二苯基取代咪唑修饰的衍生物 CB1 CB6 的 强, 因为前者的刚性更强. 通过单晶分析, 我们猜测在 香豆素 7 位取代基相同的情况下, 分子内氢键的强度和 咪唑环与香豆素环之间的二面角都会对这些化合物的 发光产生一定的影响: 氢键越强, 溶液发光效率越高; 二面角越小则导致固体发光红移. 当菲并 $[9,10-d]$ 咪唑 或者 4,5-二苯基咪唑取代位置为香豆素 6 位时, 化合物 的溶液荧光很弱(CA6 和 CB6). 因此, 在这一系列香豆 素-咪唑体系中, 取代基的供电子性、咪唑基的刚性以及 咪唑环与香豆素环之间的平面性都会对这一系列化合 物的固体发光产生重要影响.

\section{3 实验部分}

\section{1 仪器与试剂}

质谱测定使用 LCMS-IT-TOF 型质谱仪 (岛津); 核磁 测定使用 Bruker AV-400 核磁共振仪 $\left({ }^{1} \mathrm{H}: 400 \mathrm{MHz},{ }^{13} \mathrm{C}\right.$ : $100 \mathrm{MHz}, 0.5 \% \mathrm{TMS}$ 为内标); 荧光光谱测定使用 FluoroMax-4 (JONIN-YVON, HORIBA) 和 F-7000 (HITACHI) 苂光光谱仪; 紫外光谱测定使用 U-1900 (HI$\mathrm{TACHI}$ ); X-单晶衍射仪为 Xcalibur $\mathrm{E}$ (英国 Oxford 公司) 或者 Gemini (Agilent Technologies). 所用试剂和药品均 购自试剂供应商, 除特别注明外, 未经进一步处理; 溶 剂使用前都通过标准的方法进行纯化和干燥; 光谱纯有 机溶剂购自 ROE SCENTIFIC INC. 或者 DIKMA TECHNOLOGIES INC; 薄层色谱(TLC)监测通过薄层 硅胶板进行, 柱色谱分离采用青岛海洋化工厂生产的硅 胶(未加活化, 300 400 目). 化合物 $\mathbf{A - 1}{ }^{[30]} 、 \mathbf{A - 2}{ }^{[31]}$ 、 $\mathbf{A}-3^{[32]} 、 \mathbf{A}-4^{[27]} 、 \mathbf{A}-\mathbf{5}^{[27]} 、 \mathbf{A}-\mathbf{6}^{[33]}$ 参考文献合成.

\section{2 实验方法}

CA 系列和 CB 系列化合物的合成通法: 将 1 equiv. 的香豆素酫与 1.2 equiv.的 9,10-邻菲啰啉二酮或者二苯 乙二酮溶于 $10 \mathrm{~mL}$ 冰醋酸, 再加入 20 equiv.无水醋酸铵. 反应在 $120{ }^{\circ} \mathrm{C}$ 进行, TLC 监测反应完成, 时间从 $2 \mathrm{~h}$ 到 4 $\mathrm{h}$ 不等. 反应结束后, 加入水, 析出橙黄色固体, 用二氯 甲烷萃取, 合并有机相, 饱和食盐水洗两次后, 无水硫
酸钠干燥. 减压蒸除溶剂, 用 $200 \sim 300$ 目硅胶柱层析分 离, 以 $V$ (石油醚) $: V($ 乙酸乙酯 $)=8: 1$ 为洗脱剂, 得到 橙黄色固体 3-(1H-菲并[9,10- $d$ 咪唑-2-基)-2 $H$-色烯-2-酮 (CA1), 产率 67.6\%. m.p. $263 \sim 265{ }^{\circ} \mathrm{C} ;{ }^{1} \mathrm{H}$ NMR $(400$ MHz, DMSO- $\left.d_{6}\right) \delta: 13.16(\mathrm{~s}, 1 \mathrm{H}), 9.04(\mathrm{~s}, 1 \mathrm{H}), 8.91 \sim 8.76$ $(\mathrm{m}, 3 \mathrm{H}), 8.63(\mathrm{~d}, J=7.6 \mathrm{~Hz}, 1 \mathrm{H}), 8.04(\mathrm{~d}, J=6.8 \mathrm{~Hz}, 1 \mathrm{H})$, $7.80 \sim 7.60(\mathrm{~m}, 5 \mathrm{H}), 7.55(\mathrm{~d}, J=8.2 \mathrm{~Hz}, 1 \mathrm{H}), 7.46(\mathrm{t}, J=$ $7.6 \mathrm{~Hz}, 1 \mathrm{H}) ;{ }^{13} \mathrm{C}$ NMR $\left(100 \mathrm{MHz}, \mathrm{DMSO}-d_{6}\right) \delta: 159.58$, $153.57,144.23,141.87,137.23,133.08,129.90,128.42$, $128.28,127.72,127.55,126.17,126.01,125.55,124.41$, $124.25,123.30,122.76,122.42,119.60,117.69,116.61$; HRMS calcd for $\mathrm{C}_{24} \mathrm{H}_{15} \mathrm{~N}_{2} \mathrm{O}_{2}[\mathrm{M}+\mathrm{H}]^{+} 363.1128$, found 363.1179 .

3-(4,5-二苯基-1 $H$-咪唑-2-基)- $2 H$-色烯-2-酮(CB1): 产率 76.9\%. 黄色固体, m.p. $173 \sim 175{ }^{\circ} \mathrm{C} ;{ }^{1} \mathrm{H}$ NMR (400 MHz, DMSO- $\left.d_{6}\right) \delta: 12.30(\mathrm{~s}, 1 \mathrm{H}), 8.79(\mathrm{~s}, 1 \mathrm{H}), 7.97(\mathrm{~d}$, $J=7.0 \mathrm{~Hz}, 1 \mathrm{H}), 7.71 \sim 7.64(\mathrm{~m}, 1 \mathrm{H}), 7.54 \sim 7.51(\mathrm{~m}, 5 \mathrm{H})$, $7.45 \sim 7.33(\mathrm{~m}, 7 \mathrm{H}) ;{ }^{13} \mathrm{C}$ NMR (100 MHz, DMSO- $\left.d_{6}\right) \delta$ : $161.06,153.02,140.39,138.00,131.94,128.95,128.91$, $128.61,127.85,127.54,125.37,125.27,119.49,116.61$, 116.02; HRMS calcd for $\mathrm{C}_{24} \mathrm{H}_{16} \mathrm{~N}_{2} \mathrm{O}_{2} \mathrm{Na}[\mathrm{M}+\mathrm{Na}]^{+}$ 387.1104 , found 387.1108 .

7-羟基-3-(1H-菲并 $[9,10-d]$ 咪唑-2-基)- $2 H$-色烯-2-酮 (CA2): 产率 73.1\%. 黄色固体, m.p. $393 \sim 395{ }^{\circ} \mathrm{C} ;{ }^{1} \mathrm{H}$ NMR (400 MHz, DMSO- $d_{6}$ ) $\delta: 13.02$ (s, 1H), 10.89 (s, $1 \mathrm{H}), 8.95(\mathrm{~s}, 1 \mathrm{H}), 8.88 \sim 8.84(\mathrm{~m}, 2 \mathrm{H}), 8.78(\mathrm{~d}, J=7.8 \mathrm{~Hz}$, $1 \mathrm{H}), 8.61(\mathrm{~d}, J=7.6 \mathrm{~Hz}, 1 \mathrm{H}), 7.88(\mathrm{~d}, J=8.6 \mathrm{~Hz}, 1 \mathrm{H}), 7.73$ (dd, $J=16.2,8.0 \mathrm{~Hz}, 2 \mathrm{H}$ ), 7.65 (t, $J=7.6 \mathrm{~Hz}, 2 \mathrm{H}$ ), 6.93 (dd, $J=8.4,2.0 \mathrm{~Hz}, 1 \mathrm{H}), 6.89(\mathrm{~d}, J=1.8 \mathrm{~Hz}, 1 \mathrm{H}) ;{ }^{13} \mathrm{C}$ NMR $\left(100 \mathrm{MHz}\right.$, DMSO- $\left.d_{6}\right) \delta: 162.87,160.03,155.80$, $144.92,142.69,137.05,131.44,128.20,127.84,127.65$, $127.51,125.93,124.39,123.16,122.38,114.57,113.00$, 112.14, 102.51; HRMS calcd for $\mathrm{C}_{24} \mathrm{H}_{13} \mathrm{~N}_{2} \mathrm{O}_{3}[\mathrm{M}-\mathrm{H}]$ 377.0932, found 377.0921.

3-(4,5-二苯基- $1 H$-咪唑-2-基)-7-羟基- $2 H$-色烯-2-酮 (CB2): 产率 55.2\%. 黄色固体, m.p. $323 \sim 325{ }^{\circ} \mathrm{C} ;{ }^{1} \mathrm{H}$ NMR $\left(400 \mathrm{MHz}\right.$, DMSO- $\left.d_{6}\right) \delta: 12.05(\mathrm{~s}, 1 \mathrm{H}), 10.78(\mathrm{~s}$, $1 \mathrm{H}), 8.68(\mathrm{~s}, 1 \mathrm{H}), 7.79(\mathrm{~d}, J=8.6 \mathrm{~Hz}, 1 \mathrm{H}), 7.61 \sim 7.18(\mathrm{~m}$, $10 \mathrm{H}), 6.88(\mathrm{dd}, J=8.4,2.2 \mathrm{~Hz}, 1 \mathrm{H}), 6.83(\mathrm{~d}, J=2.0 \mathrm{~Hz}$, $1 \mathrm{H}) ;{ }^{13} \mathrm{C}$ NMR (100 MHz, DMSO- $\left.d_{6}\right) \delta: 162.35,160.04$, $155.40,141.25,140.41,137.78,135.33,131.15,131.05$, $129.08,128.77,128.66,128.41,128.27,127.73,127.22$, $114.44,112.88,112.12,102.45$; HRMS calcd for $\mathrm{C}_{24} \mathrm{H}_{16}$ $\mathrm{N}_{2} \mathrm{O}_{3} \mathrm{Na}[\mathrm{M}+\mathrm{Na}]^{+}$403.1053, found 403.1022.

7-丁氧基-3-(1 $H$-菲并 $[9,10-d]$ 咪唑-2-基)-2 $H$-色烯-2- 
酮(CA3): 产率 68.4\%. 黄色固体, m.p. 381 382 ${ }^{\circ} \mathrm{C} ;{ }^{1} \mathrm{H}$ NMR $\left(400 \mathrm{MHz}, \mathrm{CDCl}_{3}\right) \delta: 8.95(\mathrm{~s}, 1 \mathrm{H}), 8.90 \sim 8.70(\mathrm{~m}$, $3 \mathrm{H}), 8.61(\mathrm{~s}, 1 \mathrm{H}), 7.91(\mathrm{~d}, J=8.6 \mathrm{~Hz}, 1 \mathrm{H}), 7.82 \sim 7.65(\mathrm{~m}$, $2 \mathrm{H}), 7.64(\mathrm{t}, J=7.4 \mathrm{~Hz}, 2 \mathrm{H}), 7.10(\mathrm{~s}, 1 \mathrm{H}), 7.01(\mathrm{dd}, J=$ 8.6, $2.0 \mathrm{~Hz}, 1 \mathrm{H}), 4.09$ (t, $J=6.4 \mathrm{~Hz}, 2 \mathrm{H}), 1.83 \sim 1.64(\mathrm{~m}$, $2 \mathrm{H}), 1.52 \sim 1.32(\mathrm{~m}, 2 \mathrm{H}), 0.94(\mathrm{t}, J=7.4 \mathrm{~Hz}, 3 \mathrm{H}) ;{ }^{13} \mathrm{C}$ NMR (100 MHz, DMSO- $\left.d_{6}\right) \delta: 163.19,155.67,144.76$, 142.39 , 137.09, 131.08, 128.25, 128.19, 127.92, 127.68, $127.52,127.11,126.01,125.94,124.41,124.27,123.18$, $122.78,122.38,114.15,113.95,113.08,101.37,68.70$, 30.94 , 19.15, 14.13; HRMS calcd for $\mathrm{C}_{28} \mathrm{H}_{22} \mathrm{~N}_{2} \mathrm{O}_{3} \mathrm{Na}[\mathrm{M}+$ $\mathrm{Na}]^{+}$457.1523, found 457.1475.

7-丁氧基-3-(4,5-二苯基- $1 H$-咪唑-2-基)- $2 H$-色烯-2酮(CB3): 产率 98.2\%. 黄色固体, m.p. 134 $175{ }^{\circ} \mathrm{C} ;{ }^{1} \mathrm{H}$ NMR (400 MHz, $\left.\mathrm{CDCl}_{3}\right) \delta$ : $11.18(\mathrm{~s}, 1 \mathrm{H}), 8.86(\mathrm{~s}, 1 \mathrm{H})$, $7.74 \sim 7.34(\mathrm{~m}, 11 \mathrm{H}), 7.02 \sim 6.87(\mathrm{~m}, 2 \mathrm{H}), 4.08(\mathrm{t}, J=6.4$ $\mathrm{Hz}, 2 \mathrm{H}), 1.87 \sim 1.81(\mathrm{~m}, 2 \mathrm{H}), 1.58 \sim 1.52(\mathrm{~m}, 2 \mathrm{H}), 1.03(\mathrm{t}$, $J=7.4 \mathrm{~Hz}, 3 \mathrm{H}) ;{ }^{13} \mathrm{C} \mathrm{NMR}\left(100 \mathrm{MHz}, \mathrm{DMSO}-d_{6}\right) \delta$ : $162.77,159.93,155.27,141.08,140.08,130.71,129.14 \sim$ $127.32(\mathrm{~m}), 127.32 \sim 126.81(\mathrm{~m}), 125.64,114.03,113.76$, 113.07, 101.33, 68.63, 30.94, 19.14, 14.13; HRMS calcd for $\mathrm{C}_{28} \mathrm{H}_{24} \mathrm{~N}_{2} \mathrm{O}_{3} \mathrm{Na}[\mathrm{M}+\mathrm{Na}]^{+} 459.1679$, found 459.1681 .

7-(二乙基氨基)-3-(1H-菲并 $[9,10-d]$ 咪唑-2-基)- $2 H$ 色烯-2-酮(CA4): 产率 83.6\%. 橙红色固体, m.p. 230 $231{ }^{\circ} \mathrm{C} ;{ }^{1} \mathrm{H}$ NMR (400 MHz, DMSO- $\left.d_{6}\right) \delta: 12.85(\mathrm{~s}, 1 \mathrm{H})$, $8.88 \sim 8.84(\mathrm{~m}, 3 \mathrm{H}), 8.76(\mathrm{~d}, J=7.2 \mathrm{~Hz}, 1 \mathrm{H}), 8.61(\mathrm{~d}, J=$ $7.6 \mathrm{~Hz}, 1 \mathrm{H}), 7.78 \sim 7.51(\mathrm{~m}, 5 \mathrm{H}), 6.83(\mathrm{dd}, J=9.0,2.2 \mathrm{~Hz}$, $1 \mathrm{H}), 6.70$ (d, $J=2.2 \mathrm{~Hz}, 1 \mathrm{H}), 3.51$ (q, $J=7.0 \mathrm{~Hz}, 4 \mathrm{H}), 1.18$ $(\mathrm{t}, \quad J=7.0 \mathrm{~Hz}, 6 \mathrm{H}) ;{ }^{13} \mathrm{C}$ NMR $\left(100 \mathrm{MHz}, \mathrm{DMSO}-d_{6}\right) \delta$ : $160.61,156.75,151.80,145.71,142.64,131.09,128.05$, $127.51,125.69,124.29,110.33,109.22,108.61,96.76$, 44.73, 12.85; HRMS calcd for $\mathrm{C}_{28} \mathrm{H}_{24} \mathrm{~N}_{3} \mathrm{O}_{2}[\mathrm{M}+\mathrm{H}]^{+}$ 434.1869, found 434.1896.

7-(二乙基氨基)-3-(4,5-二苯基- $1 H$-咪唑-2-基)- $2 H$ 色烯-2-酮(CB4): 产率 99.5\%. 黄色固体, m.p. 93 $95{ }^{\circ} \mathrm{C} ;{ }^{1} \mathrm{H}$ NMR (400 MHz, DMSO- $\left.d_{6}\right) \delta: 11.87(\mathrm{~s}, 1 \mathrm{H})$, $8.59(\mathrm{~s}, 1 \mathrm{H}), 7.96 \sim 7.91(\mathrm{~m}, 1 \mathrm{H}), 7.59 \sim 7.21(\mathrm{~m}, 10 \mathrm{H})$, $6.80(\mathrm{dd}, J=9.0,2.4 \mathrm{~Hz}, 1 \mathrm{H}), 6.65(\mathrm{~d}, J=2.2 \mathrm{~Hz}, 1 \mathrm{H})$, 3.48 (q, $J=7.0 \mathrm{~Hz}, 4 \mathrm{H}), 1.16$ (t, $J=7.0 \mathrm{~Hz}, 6 \mathrm{H}) ;{ }^{13} \mathrm{C} \mathrm{NMR}$ $\left(100 \mathrm{MHz}, \mathrm{DMSO}-d_{6}\right) \delta: 160.68,156.36,151.45,141.98$, $140.52,137.52,136.02,135.47,132.69,130.71,130.04$, $129.98,129.10,128.65,128.58,128.14,127.73,127.16$, 110.25, 109.27 (s), 108.52, 96.77, 44.68, 12.81; HRMS calcd for $\mathrm{C}_{28} \mathrm{H}_{25} \mathrm{~N}_{3} \mathrm{O}_{2} \mathrm{Na}[\mathrm{M}+\mathrm{Na}]^{+} 458.1839$, found
458.1848 .

10-(1H-菲并 $[9,10-d]$ 咪唑 -2- 基 )-2,3,6,7-四氢 $1 H, 5 H, 11 H$-吡喃并 [2,3- $f$ 吡啶并 [3,2,1-ij] 喹啉-11-酮 (CA5): 产率 72.4\%. 橙红色固体, m.p. 365 366 ${ }^{\circ} \mathrm{C} ;{ }^{1} \mathrm{H}$ NMR (400 MHz, $\left.\mathrm{CDCl}_{3}\right) \delta: 11.97(\mathrm{~s}, 1 \mathrm{H}), 8.91(\mathrm{~s}, 1 \mathrm{H})$, $8.79 \sim 8.72(\mathrm{~m}, 3 \mathrm{H}), 8.15(\mathrm{~d}, J=7.6 \mathrm{~Hz}, 1 \mathrm{H}), 7.72 \sim 7.65$ $(\mathrm{m}, 4 \mathrm{H}), 7.09(\mathrm{~s}, 1 \mathrm{H}), 3.36 \sim 3.22(\mathrm{~m}, 4 \mathrm{H}), 2.97(\mathrm{t}, J=6.4$ $\mathrm{Hz}, 2 \mathrm{H}), 2.82(\mathrm{t}, J=6.2 \mathrm{~Hz}, 2 \mathrm{H}), 2.08 \sim 1.98(\mathrm{~m}, 4 \mathrm{H}) ;{ }^{13} \mathrm{C}$ NMR $\left(100 \mathrm{MHz}, \mathrm{CDCl}_{3}\right) \delta: 162.61,151.53,147.05$, $145.85,141.33,128.34,127.04,126.84,126.29,126.05$, $125.19,123.96,123.33,122.79,122.44,121.06,120.62$, 119.68, 108.84, 107.42, 106.14, 50.19, 49.78, 27.55, 21.27, 20.35, 20.23; HRMS calcd for $\mathrm{C}_{30} \mathrm{H}_{24} \mathrm{~N}_{3} \mathrm{O}_{2}[\mathrm{M}+\mathrm{H}]^{+}$ 458.1863 , found 458.1865 .

10-(4,5-二苯基 - $1 H$ - 咪唑 -2- 基 )-2,3,6,7-四氢 $1 H, 5 H, 11 H$ - 吡喃并 [2,3- $f$ 吡啶并 [3,2,1-ij] 喹啉-11-酮 (CB5): 产率 48.6\%. 黄色固体, m.p. 276 277 ${ }^{\circ} \mathrm{C} ;{ }^{1} \mathrm{H}$ NMR (400 MHz, $\left.\mathrm{CDCl}_{3}\right) \delta: 11.22(\mathrm{~s}, 1 \mathrm{H}), 8.68(\mathrm{~s}, 1 \mathrm{H})$, 7.69 (d, $J=6.8 \mathrm{~Hz}, 2 \mathrm{H}), 7.51(\mathrm{~d}, J=6.8 \mathrm{~Hz}, 2 \mathrm{H}), 7.42 \sim$ $7.35(\mathrm{~m}, 6 \mathrm{H}), 7.04(\mathrm{~s}, 1 \mathrm{H}), 3.34 \sim 3.32(\mathrm{~m}, 4 \mathrm{H}), 2.97(\mathrm{t}$, $J=6.4 \mathrm{~Hz}, 2 \mathrm{H}), 2.82(\mathrm{t}, J=6.2 \mathrm{~Hz}, 2 \mathrm{H}), 2.11 \sim 1.95(\mathrm{~m}$, $4 \mathrm{H}) ;{ }^{13} \mathrm{C} \mathrm{NMR}\left(100 \mathrm{MHz}, \mathrm{CDCl}_{3}\right) \delta: 162.35,151.22$, $146.60,142.40,139.57,128.80,128.35,127.97,127.63$, $126.92,125.84,119.52,108.82,107.71,106.20$, 50.15, 49.74, 27.56, 21.34, 20.43, 20.2; HRMS calcd for $\mathrm{C}_{30} \mathrm{H}_{25} \mathrm{~N}_{3} \mathrm{O}_{2} \mathrm{Na}[\mathrm{M}+\mathrm{Na}]^{+} 482.1839$, found 482.1779 .

6-(1H-菲并 $[9,10-d]$ 咪唑-2-基)-2 $H$-色烯-2-酮(CA6): 产率 58.5\%. 淡黄色固体, m.p. 406 407 ${ }^{\circ} \mathrm{C} ;{ }^{1} \mathrm{H}$ NMR $\left(400 \mathrm{MHz}, \mathrm{DMSO}-d_{6}\right) \delta: 8.87(\mathrm{~d}, J=8.2 \mathrm{~Hz}, 2 \mathrm{H}), 8.62$ (d, $J=2.0 \mathrm{~Hz}, 1 \mathrm{H}), 8.57(\mathrm{~d}, J=7.8 \mathrm{~Hz}, 2 \mathrm{H}), 8.51(\mathrm{dd}, J=8.6$, $2.0 \mathrm{~Hz}, 1 \mathrm{H}), 8.26(\mathrm{~d}, J=9.6 \mathrm{~Hz}, 1 \mathrm{H}), 7.76(\mathrm{t}, \quad J=7.2 \mathrm{~Hz}$, $2 \mathrm{H}), 7.71 \sim 7.61(\mathrm{~m}, 3 \mathrm{H}), 6.61(\mathrm{~d}, J=9.6 \mathrm{~Hz}, 1 \mathrm{H}) ;{ }^{13} \mathrm{C}$ NMR $\left(100 \mathrm{MHz}, \mathrm{DMSO}-d_{6}\right) \delta$ : 159.68, 153.86, 147.84, $144.17,129.47,127.66,127.14,126.88,125.93,125.34$, 123.94, 121.86, 119.20, 117.04, 116.98; HRMS calcd for $\mathrm{C}_{24} \mathrm{H}_{13} \mathrm{~N}_{2} \mathrm{O}_{2}[\mathrm{M}-\mathrm{H}]^{-}$361.0983, found 361.1006.

6-(4,5-二苯基- $1 H$-咪唑-2-基)-2 $H$-色烯-2-酮(CB6): 产率 91.2\%. 淡黄色固体, m.p. 267 268 ${ }^{\circ} \mathrm{C} ;{ }^{1} \mathrm{H}$ NMR $\left(400 \mathrm{MHz}, \mathrm{DMSO}-d_{6}\right) \delta: 12.84(\mathrm{~s}, 1 \mathrm{H}), 8.42(\mathrm{~s}, 1 \mathrm{H}), 8.30$ $(\mathrm{d}, J=8.6 \mathrm{~Hz}, 1 \mathrm{H}), 8.17(\mathrm{~d}, J=9.6 \mathrm{~Hz}, 1 \mathrm{H}), 7.66 \sim 7.05$ $(\mathrm{m}, 11 \mathrm{H}), 6.57(\mathrm{~d}, J=9.6 \mathrm{~Hz}, 1 \mathrm{H}) ;{ }^{13} \mathrm{C} \mathrm{NMR}(100 \mathrm{MHz}$, DMSO- $\left.d_{6}\right) \delta: 160.26,153.73,144.73,137.75,135.44$, $131.38,129.17,129.06,128.97,128.84,128.68,128.34$, $127.56,127.35,127.10,125.33,119.50,117.30$; HRMS 
calcd for $\mathrm{C}_{24} \mathrm{H}_{17} \mathrm{~N}_{2} \mathrm{O}_{2}[\mathrm{M}+\mathrm{H}]^{+}$365.1285, found 365.1318 .

\section{辅助材料(Supporting Information) 部分化合物 CA1、} $\mathrm{CA4} 、 \mathrm{CB1} 、 \mathrm{CB3} \sim \mathrm{CB5}$ 的单晶数据以及系列染料化合 物 CA1 CA5 及 $\mathrm{CB1} \sim \mathrm{CB} 6$ 的核磁谱图. 这些材料可 以免费从本刊网站(http://sioc-journal.cn/)上下载.

\section{References}

[1] Mei, J.; Hong, Y.; Lam, J. W. Y.; Qin, A.; Tang, Y.; Tang, B. Z. Adv. Mater. 2014, 26, 5429.

[2] Shimizu, M.; Hiyama, T. Chem. Asian J. 2010, 5, 1516.

[3] Qian, J.; Tang, B. Z. Chem 2017, 3, 56.

[4] Xia, Z.; Shao, A.; Li, Q.; Zhu, S.; Zhu, W. Acta Chim. Sinica 2016, 74, 351 (in Chinese).

(夏志清, 邵安东, 李强, 朱世琴, 朱为宏, 化学学报, 2016, 74, 351.)

[5] Shimizu, M.; Kaki, R.; Takeda, Y.; Hiyama, T.; Nagai, N.; Yamagishi, H.; Furutani, H. Angew. Chem., Int. Ed. 2012, 51, 4095.

[6] Yuan, C.; Saito, S.; Yusa, H.; Camacho, C.; Irle, S.; Hisaki, I.; Yamaguchi, S. J. Am. Chem. Soc. 2013, 135, 8842.

[7] Matsui, M.; Ooiwa, K.; Okada, A.; Kubota, Y.; Funabiki, K.; Sato, H. Dyes Pigm. 2013, 99, 916.

[8] Marwitz, A. J. V.; Jenkins, J. T.; Zakharov, L. N.; Liu, S.-Y. Angew. Chem., Int. Ed. 2010, 49, 7444.

[9] Luo, X.; Li, J.; Li, C.; Heng, L.; Dong, Y. Q.; Liu, Z.; Bo, Z.; Tang, B. Z. Adv. Mater. 2011, 23, 3261.

[10] Kwon, M. S.; Gierschner, J.; Yoon, S.-J.; Park, S. Y. Adv. Mater. 2012, 24, 5487.

[11] Ren, T.-B.; Xu, W.; Zhang, W.; Zhang, X.-X.; Wang, Z.-Y.; Xiang, Z.; Yuan, L.; Zhang, X.-B. J. Am. Chem. Soc. 2018, 140, 7716.

[12] Zhao, D.; Li, G.; Wu, D.; Qin, X.; Neuhaus, P.; Cheng, Y.; Yang, S.; Lu, Z.; Pu, X.; Long, C.; You, J. Angew. Chem., Int. Ed. 2013, 52, 13676.

[13] Wang, S.; Xiao, S.; Chen, X.; Zhang, R.; Cao, Q.; Zou, K. Dyes Pigm. 2013, 99, 543.
[14] Kubota, Y.; Hara H.; Tanaka, S.; Funabiki, K.; Matsui, M. Org. Lett. 2011, 13, 6544.

[15] Kubota, Y.; Tsuzuki, T.; Funabiki, K.; Ebihara, M.; Matsui, M. Org. Lett. 2010, 12, 4010.

[16] Kubota, Y.; Ozaki, Y.; Funabiki, K.; Matsui, M. J. Org. Chem. 2013, 78, 7058.

[17] Li, W.; Lin, W.; Wang, J.; Guan, X. Org. Lett. 2013, 15, 1768.

[18] Chen, P.-Z.; Zhang, H.; Niu, L.-Y.; Zhang, Y.; Chen, Y.-Z.; Fu, H.-B.; Yang, Q.-Z. Adv. Funct. Mater. 2017, 27, 1700332.

[19] Liu, N.; Chen, P.-Z.; Wang, J.-X.; Niu, L.-Y.; Yang, Q.-Z. Chin. Chem. Lett. 2019, 30, 1939.

[20] Zheng, M.; Sun, M.; Li, Y.; Wang, J.; Bu, L.; Xue, S.; Yang, W. Dyes Pigm. 2014, 102, 29.

[21] Zhang, Z.; Zhang, Y.; Yao, D.; Bi, H.; Javed, I.; Fan, Y.; Zhang, H.; Wang, Y. Cryst. Growth Des. 2009, 9, 5069.

[22] Dong, Y.; Xu, B.; Zhang, J.; Tan, X.; Wang, L.; Chen, J.; Lv, H.; Wen, S.; Li, B.; Ye, L.; Zou, B.; Tian, W. Angew. Chem., Int. Ed. 2012, 51, 10782.

[23] Chen, B.; Yu, G.; Li, X.; Ding, Y.; Wang, C.; Liu, Z.; Xie, Y. J. Mater. Chem. C 2013, 1, 7409.

[24] Xie, L.; Chen, Y.; Wu, W.; Guo, H.; Zhao, J.; Yu, X. Dyes Pigm. 2012, 92, 1361.

[25] Huang, D.; Chen, Y.; Zhao, J. Dyes Pigm. 2012, 95, 732.

[26] Park, S.-Y.; Ebihara, M.; Kubota, Y.; Funabiki, K.; Matsui, M. Dyes Pigm. 2009, 82, 258.

[27] Hou, J.-T.; Yang, J.; Li, K.; Liao, Y.-X.; Yu, K.-K.; Xie, Y.-M.; Yu, X.-Q. Chem. Commun. 2014, 50, 9947.

[28] Hou, J.-T.; Li, K.; Qin, C.; Yu, X.-Q. Sci. Sin. Chim. 2019, 49, 346 (in Chinese). (后际挺，李坤，覃彩芹，余孝其，中国科学：化学，2019，49, 346.)

[29] Hou, J.-T.; Kim, H. S.; Duan, C.; Ji, M. S.; Wang, S.; Zeng, L.; Ren, W. X.; Kim, J. S.; Yu, X.-Q. Chem. Commun. 2019, 55, 2533.

[30] Pottie, I R.; Nandaluru, P. R.; Benoit, W. L.; Miller, D. O.; Dawe, L. N.; Bodwell, G. J. J. Org. Chem. 2011, 76, 9015.

[31] Hou, J.-T.; Li, K.; Liu, B.-Y.; Liao, Y.-X.; Yu, X.-Q. Tetrahedron 2013, 69, 2118.

[32] Lin, W.; Yuan, L.; Feng, J.; Cao, X. Eur. J. Org. Chem. 2008, 2689.

[33] Xu, S.; Xu, S.; Li, L. Acta Pharm. Sin. 2000, 35, 103 (in Chinese). (徐嵩, 徐世平, 李兰敏, 药学学报, 2000, 35, 103.) 